\title{
The neural substrates of response inhibition to negative information across explicit and implicit tasks in GAD patients: electrophysiological evidence from an ERP study
}

\section{OPEN ACCESS}

Edited by:

Narayanan Srinivasan,

University of Allahabad, India

Reviewed by:

Adrian Von Muhlenen,

University of Warwick, UK

David Vaughn Becker,

Arizona State University, USA

Mark Fenske

University of Guelph, Canada

${ }^{*}$ Correspondence:

Kai Wang,

Laboratory of Cognitive

Neuropsychology, Department of

Medical Psychology, Anhui Medical

University, Hefei 230032, China

wangkai1964@126.com;

Xinlong $\mathrm{Hu}$,

Anhui Mental Health Center,

Hefei 230032, China

904484245@qq.com

${ }^{\dagger}$ These authors have contributed equally to this work.

Specialty section:

This article was submitted to Cognitive Science, a section of the

journal Frontiers in Psychology

Received: 07 August 2014

Accepted: 25 February 2015

Published: 20 March 2015

Citation:

Yu F, Zhu C, Zhang L, Chen X, Li D,

Zhang L, Ye R, Dong Y, Luo Y, Hu X

and Wang $K$ (2015) The neural

substrates of response inhibition to negative information across explicit

and implicit tasks in GAD patients: electrophysiological evidence from an

ERP study.

Front. Psychol. 6:275.

doi: 10.3389/fpsyg.2015.00275
Fengqiong $\mathrm{Yu}^{1+}$, Chunyan Zhu ${ }^{1+}$, Lei Zhang ${ }^{1}$, Xingui Chen ${ }^{2}$, Dan Li ${ }^{2}$, Long Zhang ${ }^{2}$, Rong Ye ${ }^{1}$, Yi Dong ${ }^{3}$, Yuejia $\mathrm{Luo}^{4}$, Xinlong Hu${ }^{3 *}$ and Kai Wang ${ }^{1,2 *}$

${ }^{1}$ Laboratory of Cognitive Neuropsychology, Department of Medical Psychology, Anhui Medical University, Hefei, China, ${ }^{2}$ Department of Neurology, The First Affiliated Hospital of Anhui Medical University, Hefei, China, ${ }^{3}$ Anhui Mental Health Center, Hefei, China, ${ }^{4}$ Institute of Social and affective Neuroscience, Shenzhen University, Shenzhen, China

Background: It has been established that the inability to inhibit a response to negative stimuli is the genesis of anxiety. However, the neural substrates of response inhibition to sad faces across explicit and implicit tasks in general anxiety disorder (GAD) patients remain unclear.

Methods: Electrophysiological data were recorded when subjects performed two modified emotional go/no-go tasks in which neutral and sad faces were presented: one task was explicit (emotion categorization), and the other task was implicit (gender categorization).

Results: In the explicit task, electrophysiological evidence showed decreased amplitudes of no-go/go difference waves at the N2 interval in the GAD group compared to the control group. However, in the implicit task, the amplitudes of no-go/go difference waves at the N2 interval showed a reversed trend. Source localization analysis on nogo/N2 components revealed a decreased current source density (CSD) in the right dorsal lateral prefrontal cortex in GAD individuals relative to controls. In the implicit task, the left superior temporal gyrus and the left inferior parietal lobe showed enhanced activation in GAD individuals and may compensate for the dysfunction of the right dorsal lateral prefrontal cortex.

Conclusion: These findings indicated that the processing of response inhibition to socially sad faces in GAD individuals was interrupted in the explicit task. However, this processing was preserved in the implicit task. The neural substrates of response inhibition to sad faces were dissociated between implicit and explicit tasks.

Keywords: general anxiety disorder, go/no-go task, response inhibition, N2, source localization

\section{Introduction}

General anxiety disorder (GAD) is the most persistent, severe, and prevalent class of mental disorder. This disorder is characterized by excessive worry, anxiety, and tension in addition to other somatic symptoms (Wittchen, 2002). Some studies have shown that anxiety is 
often associated with impaired cognitive control and avoidance behavior (Sehlmeyer et al., 2010). Response inhibition is an important component of this cognitive control system. Indeed, it has been established that an inability to inhibit a response to negative stimuli is linked to anxiety (Pacheco-Unguetti et al., 2012).

Response inhibition refers to inhibiting inappropriate behavior according to current task demands (Sehlmeyer et al., 2010). The typical paradigm of response inhibition is a go/no-go task. The different cognitive processing elements of response inhibition are conflict monitoring and action inhibition. Event-related brain potentials (ERPs), from recording electroencephalograms time-locked to stimuli, are a non-invasive brain imaging method. The main advantage of this method is high time resolution and thus can manifest the time course of brain activity. The methods have been frequently used to explore the neural substrates of response inhibition (Fabiani et al., 2000). It is believed that nogo-related N2 is related to conflict-monitoring processes, while no-go-related P3 is related to conflict resolution and behavior inhibition (Donkers and van Boxtel, 2004; Kropotov et al., 2011). Many neuroimaging studies have indicated that the right inferior prefrontal cortex (rIFC) plays a crucial role in the neural substrate of response inhibition to emotional stimuli (Ochsner et al., 2004; Shafritz et al., 2006; Goldstein et al., 2007; Berkman et al., 2009; Padmala and Pessoa, 2010). Previous studies have reported that anxious individuals exhibit a response inhibition deficit. An ERP study in children that used a modified emotional go/no-go task detected that when asked to suppress facial stimuli, anxious children showed decreased no-go/go differences in wave amplitudes during the N2 stage, and no-go/N2 amplitudes for calm faces predicted self-reported anxiety levels (Hum et al., 2013). Another study found that no-go/N2 was negatively related to anxietyrelated personality traits (Sehlmeyer et al., 2010). These results suggested that dysfunctional response inhibition may be a trait indicator of anxiety. Although anxiety appears to disrupt inhibition, the neural correlates of this effect are far less understood. For example, anxiety may be due to a reduced ability to monitor conflict or may equally be linked to a reduced ability to apply active inhibition (Botvinick et al., 2001; Aarts and Pourtois, 2010; Berggren and Derakshan, 2013).

According to previous studies, behavioral and neural evidence confirmed that anxious individuals show an attention bias to negative faces, which results in greater negative emotional impulse strength and negative expressivity and reactivity to their emotions (Semlitsch et al., 1986; Decker et al., 2008; Roemer et al., 2009). The high arousal of negative emotions critically interrupts cognitive function, such as attention, memory, decision-making, executive function, and response inhibition ability (Salters-Pedneault et al., 2006; Mantella et al., 2007; Sass et al., 2010; Sehlmeyer et al., 2010; Paulus and Yu, 2012). Negative attention bias refers to the act of automatically fixing attention to negative aspects of internal or external events. ERP studies have reported shorter latency and larger amplitudes of P1, which were generated by extrastriate visual areas and index early attention processing in anxious compared to control individuals (Vuilleumier and Pourtois, 2007; Peschard et al., 2013). Another early ERP component that relates to top-down attention resources in face processing are N170 and the vertex positive potential (VPP; Ofan et al., 2013; Peschard et al., 2013). It has been reported that anxious individuals exhibit enhanced N170 and VPP activity when processing negative facial expressions (Frenkel and Bar-Haim, 2011; Ofan et al., 2013).

Sad facial expressions are one kind of fundamental negative emotional stimuli that convey important information in social communications (Schneider et al., 1994; Luo et al., 2010). Emotions induced by sad facial expressions influence an individual's ability to inhibit inappropriate behavior. Many psychiatric individuals showed disabilities when regulating the relationship between sad facial information and response inhibition (Gehricke and Shapiro, 2000; Dziobek et al., 2011; Duerden et al., 2013; Hummer et al., 2013). When new mothers had more sad expressions, their infants expressed less joy and spent more time in joint negative affective states (Termine and Izard, 2009). Indeed, sad emotion is also associated with anxiety traits. When presented with sad facial stimuli, general social anxiety disorder patients showed hyperactivity in the medial frontal cortex extending into the anterior cingulated cortex (Labuschagne et al., 2012). However, few studies have focused on the relationship between sad emotion and anxiety.

In social situations, effortful explicit interpretation of the meaning of facial expressions may be required to guide an individual's social responses. Yet, in familiar situations, facial expression encoding implicitly may also affect behavior without full cognitive awareness. Explicit processing means that facial expression is within the voluntary attention scope and is directly processed, whereas implicit processing means that facial expression is within the involuntary attention scope, and therefore is incidentally processed. Thus, the attention resource for stimuli processing is distinct between the two conditions. In fact, implicit and explicit facial processing serve different functions (Taylor et al., 2003) and have distinct neural substrates (Winston et al., 2003; Linden et al., 2010; Valdes-Conroy et al., 2014). It has been confirmed that facial expression processed explicitly and implicitly induced distinct emotional intensity in subject reports. Rating pictures was associated with significantly less intensity of sadness than passively viewing pictures, likely because the rating task reduced the activation of related brain regions responsible for an emotional experience (Taylor et al., 2003). Thus, faces processed implicitly and explicitly may showed dissociated effects on response inhibition. Our previous studies revealed that response inhibition was modulated by sad facial information at the action inhibition stage when facial expressions were processed explicitly rather than implicitly (Yu et al., 2014). However, until now, whether GAD patients show a deficit in response inhibition to sad faces across implicit and explicit conditions remains unclear.

The aim of the present study was to investigate the neural substrates of response inhibition to sad faces in GAD individuals across explicit and implicit tasks in a time course using ERP methods. We developed a modified emotional go/no-go task. In the task-relevant situation, subjects make their go/nogo decision according to the recognition of emotional categories; i.e., the emotional information was explicitly processed. 
In the task-irrelevant situation, subjects respond or inhibit their response based on the identification of the gender of the face, i.e., the emotional processing was implicit. Furthermore, in the explicit or implicit tasks, we used the same set of stimulus to preclude the distractions due to additional stimulus. A combination of ERP and source localization methods was used in order to further characterize the temporal and spatial characteristics of emotional response inhibition in implicit and explicit tasks in the GAD compared to normal groups. Based on previous studies indicating that GAD individuals exhibit attention bias to negative stimuli and dysfunction of response inhibition to negative stimuli, we hypothesized GAD individuals would show shorter latency and larger amplitudes in early ERP components, such as P1, N170, and VPP. More importantly, GAD individuals showed poor behavior performance and abnormal N2 and P3 components, as well as inhibition-related brain areas, such as the rIFC, in both implicit and explicit tasks.

\section{Materials and Methods}

\section{Participants}

Thirty-eight participants took part in the present experiment. Nineteen right-handed adults (nine female) aged $30.3 \pm 7.3$ (mean \pm SD) years participated in the study from the Anxiety Disorders Clinic in Anhui Mental Health Center. Diagnostic assessments were completed by two psychiatrists according to DSM-IV. All GAD patients scored above 14 using the Hamilton Anxiety Rating Scale [HAMA-14]. The exclusion criteria included: (a) an IQ less than 85, according to the Wechsler Abbreviated Scale of Intelligence (WASI); (b) a current or lifetime history of psychotic disorder, bipolar disorder, pervasive developmental disorder, substance abuse, or eating disorder; (c) concurrent behavioral or drug treatments for anxiety; and (d) chronic medical illness that required a daily medication regime. Twenty age-matched adults screened for current and past psychiatric and neurological disorders were recruited through local advertisements. One woman in the control group was excluded from the analysis due to poor recording quality, leaving 19 for the final analysis (nine females). The matched group scored within the normal range on the HAMA-14. All participants had normal or corrected-to-normal vision. Table 1 presents the background tests and characteristics of the final sample of clinical and comparison groups. All participants signed an informed consent form for the experiment. This study was approved by The Ethics Committee of Anhui Medical University.

\section{Stimuli}

We selected 40 sad and 40 neutral faces from the native Chinese Facial Affective Picture System, including 20 female and 20 male faces displaying each emotion type. The faces in the Chinese Facial Affective Picture System were assessed with a 9-point scale by 100 college students from two colleges in Beijing and have been used in other studies (Luo et al., 2010). The stimulus for the present experiment were selected in such a way that they differed significantly in valence from one another
TABLE 1 | Group characteristics of the GAD group and CON group.

\begin{tabular}{llll}
\hline & GAD group & CON group & $\begin{array}{c}\text { Between group } \\
\text { comparison }\end{array}$ \\
\cline { 2 - 4 } & Mean (SD) & Mean (SD) & P-value \\
\hline Sex (male/female) & $9 / 10$ & $9 / 10$ & 0.87 \\
Age (years) & $30.3(7.3)$ & $26.6(7.6)$ & 0.13 \\
Education (years) & $12.9(3.4)$ & $13.5(1.9)$ & 0.49 \\
Handedness (R/L) & $19 / 0$ & $20 / 1$ & 0.32 \\
HAMA & $25.4(7.1)$ & $6.1(1.2)$ & $<0.001$
\end{tabular}

GAD, general anxiety disorder; CON, control; HAMA, Hamilton Anxiety Rating Scale.

$(t=11.65, P<0.001[M \pm \mathrm{SD}$, sad: $3.11 \pm 0.63$, neutral: $4.49 \pm 0.41])$, but were similar in arousal $(P>0.5)$. The stimulus were similar to one another in size, background, spatial frequency, contrast grade, brightness, and other physical properties. Each picture was cropped into the shape of an ellipse that incorporated the facial characteristics using Adobe Photoshop $8.0^{\circledR}$ software. The screen resolution was 72 pixels per inch, and the viewing angle was $5.7 \times 4.6^{\circ}$. The subjects were seated in a soundproof room with their eyes approximately $100 \mathrm{~cm}$ from a 17 -in screen. All stimuli were displayed in the center of the screen.

\section{Experimental Procedures}

The experimental procedure was similar to that of previous studies (Yu et al., 2014). The present experiment included two types of emotional go/no-go paradigms: implicit task and explicit task. During the implicit task, the participants were instructed to respond after the presentation of faces depicting one gender (go trials) and to give no response after the other gender (no-go trial). In the explicit task, we asked the participants to respond or inhibit their behavior according to the valence of the facial expression. The implicit task and explicit task were presented in two separate parts, and the order of the two parts was counterbalanced across participants. Furthermore, each parts was sub-divided into two blocks in which the facial stimuli were counterbalanced in terms of whether they indicated go or nogo trials. Thus, the experimental procedure included four blocks that is negative-go/neutral-nogo and neutral-go/negative-nogo in explicit task, female-go/male-nogo, and male-go/female-nogo in implicit task.

Each block consisted of 480 trials that include 144 no-go stimuli and 336 go stimuli (30\% vs. 70\%). In each block, the go and no-go stimuli were presented pseudo-randomly, and a no-go trial was always preceded by a go trial. This was done in order to induce pre-potent motor responses and obvious conflict during response inhibition. At the start of each block, an instruction screen was presented for $5 \mathrm{~min}$ that prompted the participants to press or refrain from pressing the "J" key with their right hand according to the facial expression or gender.

Each trial was initiated by a small gray cross that was displayed for a variable duration (200-400 ms) on the black background. An emotional face appeared at the center of the screen for $1,000 \mathrm{~ms}$. The participants were instructed to 
respond as quickly as possible on the promise of accuracy after the face was presented. Each response was followed by a blank screen presented for 1,200 to $1,500 \mathrm{~ms}$, before the next trial began. The experimental procedure is presented in Figure 1. A training session including 20 trials was incorporated before the formal experiment. The stimuli included in the training session were different from those used in the main experiment. We compiled and executed all programs using E-Prime software (Psychology Software Tools, Inc., Pittsburgh, PA, USA). The independent variables of the present experiment were task (explicit and implicit), emotion (sad and neutral), trial type (go and no-go), and group (GAD and control). We used a multivariate analysis of variance for data analysis.

\section{Event-Related Potential Recording}

Electroencephalography (EEG) was recorded from 64 scalp sites using tin electrodes mounted on an elastic cap (Neuro Scan, Sterling, VA, USA) according to the international 10/20 system. The system was grounded with a forehead electrode. All EEG signals were referenced to the left mastoid and were re-referenced off-line to the average of the left and right mastoids. Vertical electro-oculogram (EOG) data were recorded supraorbitally and infraorbitally in the left eye. Horizontal EOG data were recorded as the left versus right orbital rim. EEG and EOG activity was amplified with a $0.01-100 \mathrm{~Hz}$ band-pass filter and continuously sampled at $500 \mathrm{~Hz} / \mathrm{channel}$. All electrode impedances were maintained below $5 \mathrm{k} \Omega$. Ocular artifacts were removed from the EEG signals using a regression procedure implemented in Neuroscan software (Semlitsch et al., 1986). Trials with remaining EOG artifacts, amplifier clipping artifacts, or peak-to-peak deflections exceeding $\pm 100 \mu \mathrm{V}$ were excluded from averaging.
The EEG activities during correct responses in each condition were aligned and averaged separately. The ERP waveforms were time-locked to the onset of the face stimuli, and the average epoch was 1,200 ms, including a $200 \mathrm{~ms}$ pre-stimulus baseline.

\section{Behavior Result Analysis}

We used signal detection theory to analyze the behavioral results. Signal detection theory is a method that discerns signal from noise. It assumes that the perceiver has a distribution of internal responses for both signal and noise (Snodgrass and Corwin, 1988; Stanislaw and Todorov, 1999). Based on this theory, we investigated participant's discrimination accuracy (distance between signal and noise distributions) and decision bias (tendency to respond 'signal' or 'noise'). The discrimination accuracy $d^{\prime}$ was defined as $d^{\prime}=\left(\mu_{s}-\mu_{n}\right) / \sigma$, where $\mu_{s}$ is the mean of signal distribution and $\mu_{\mathrm{n}}$ is the mean of noise distribution, and $\sigma$ is the common standard deviation of both distributions. It is calculated as $d^{\prime}=Z_{\text {hit rate }}-Z_{\text {false alarm rate }}$, with four possible outcomes: hit (signal present and subject's response is 'yes'), miss (signal present and subject's response is 'no'), false alarm (signal absent and subject's response is 'yes'), and correct rejection (signal absent and subject's response is 'no'). Decision bias $\beta$ was defined as $\beta=f_{s}(\lambda) / f_{n}(\lambda)$, where $f_{s}(\lambda)$ is the height of the signal distribution at a given criterion $\lambda$ and $f_{n}(\lambda)$ is the height of the noise distribution at the same $\lambda$. It was calculated as $\beta=\exp$ $\left(d^{\prime} \times C\right)$, where $C=-\left(\mathrm{Z}_{\text {hit rate }}+\mathrm{Z}_{\text {false alarm rate }}\right) / 2$. Because the data set does not meet the Gaussian distribution and all comparisons were based on an a priori hypothesis for small samples, we used non-parametric tests. We used two independent sample tests to compare the $\mathrm{d}^{\prime}$ and $\beta$ variables between the two groups.
A

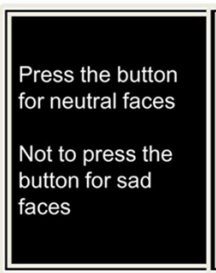

Explicit task (emotion recognition)

B

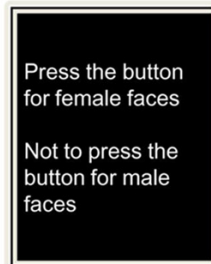

Implicit task (gender identification)
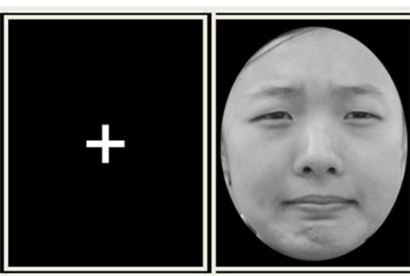

Nogo
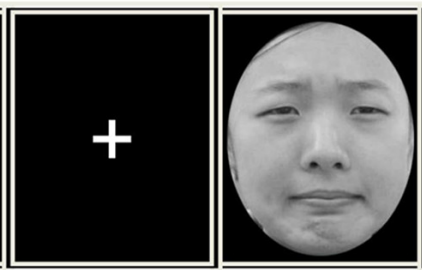

Go

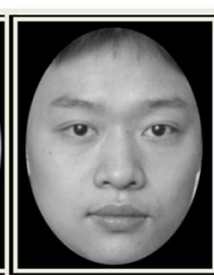

Go

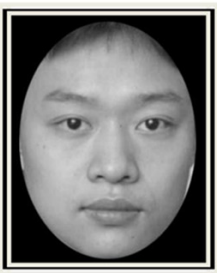

Nogo

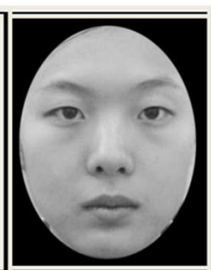

Go

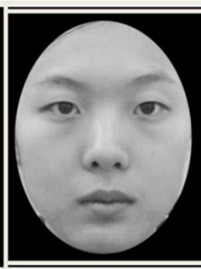

Go

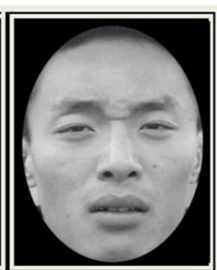

Nogo

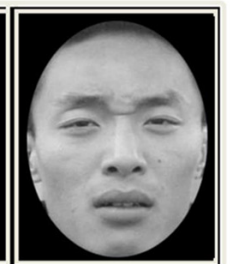

Nogo

FIGURE 1 | Trial design for (A) an explicit and (B) an implicit emotional Go/No-go tasks. In explicit task, subjects pressed a response button or inhibit their behavior according to the facial expression (sad/neutral). While in implicit task, subjects made their motor actions based on the facial gender (male/female). 


\section{ERP Measure and Analysis}

In this study, P1, VPP, N170, N2, and P3 components were measured and the peak and latency (P1, VPP, N170, N2, and P3: from stimulus onset to the peak of each component) and amplitudes (P1, VPP, and N170: baseline to peak; N2 and P3: mean amplitudes) were analyzed. According to the topographical distribution of grand-averaged ERP activity and previous studies (Williams et al., 2006; Righart and de Gelder, 2007; Yuan et al., 2008; Yu et al., 2009), different sets of electrodes sites were chosen for these components. We selected the following nine electrode sites for statistical analysis of the VPP (120-220 ms), N2 (220$320 \mathrm{~ms}$ ), and P3 components (450-550 $\mathrm{ms}$ and 550-650 ms): F3, C3, P3, Fz, Cz, Pz, F4, C4, and P4. The P7, PO7, P8, and PO8 sites were used for statistical analysis of N170 (120$220 \mathrm{~ms}$ ), and $\mathrm{O} 1, \mathrm{OZ}$, and $\mathrm{O} 2$ were used for statistical analysis of P1 (60-140 ms). A 5-way mixed design ANOVA on the amplitude and latency of each component was conducted with task (two levels: implicit, explicit), emotion (two levels: neutral, sad), trial type (two levels: go and no-go) and electrode as within subject factors, and group (anxiety and control) as between subject factors. $P$-values were corrected by GreenhouseGeisser correction. We used a Bonferroni method for multiple comparisons.

\section{Source-Localization Analysis}

The sLORETA analysis was applied to calculate the cerebral generators of response inhibition (Pascual-Marqui, 2002). sLORETA is a three-dimensional discrete linear solution that has been frequently used for EEG source analysis. sLORETA is used to estimate current density distributions restricted to the cortical gray matter and the hippocampus in the digitized MNI atlas with 6,239 voxels at a spatial resolution of $5 \mathrm{~mm}$. The sLORETA method has been shown to produce results that coincide with those provided by other brain imaging methods using equivalent paradigms (Dierks et al., 2000; Vitacco et al., 2002; Mulert et al., 2004; Pizzagalli et al., 2004).

To identify the different neural responses of response inhibition between the anxiety and control groups, we compared voxel-based whole brain sLORETA images between the groups during the no-go condition based on statistical non-parametric mapping (SnPM) methodology (Nichols and Holmes, 2002).

\section{Results}

\section{Behavioral Performance}

The response accuracy and response time were presented in Table 2. We used signal detection theory to analyze response accuracy. The ANOVA analysis revealed that GAD patients had significantly smaller $d^{\prime}$ values compared to the control group when asked to respond to negative faces and inhibited responses to neutral faces under the explicit task $(t=2.82$, $P<0.05)$, indicating diminished ability under this task. The differences in $d^{\prime}$ between the two groups did not reach significance in other conditions. We did not observe a significant difference in $\beta$ values between the two groups in any condition. It is worth noting that $d^{\prime}$ and $\beta$ are two independent measures; that is, discrimination accuracy does not correlate with decision bias. Our results demonstrated significant impairment in discrimination accuracy in response to negative faces and inhibited responses to neutral faces indexed by $d^{\prime}$, yet there was no significant deficit in likelihood ratio decision bias measured by $\beta$ under the explicit task. In addition, the statistical analysis of $d^{\prime}$ and $\beta$ showed that the difference between the implicit task and explicit task were not significant in both groups.

The repeated-measures ANOVA showed that the reaction times undergo conditions were significantly affected by task and emotion $\left(F_{1,36}=10.28, P<0.005 ; F_{1,36}=13.59, P<0.005\right)$. The reaction times were significantly shorter for the explicit task than for the implicit task and were shorter under negative conditions than under neutral conditions. Additionally, the three-way interaction was also significant $\left(F_{1,36}=5.95\right.$, $P<0.05)$. We further explored the 3-way interaction effect by splitting the task factor into two separate 2-way ANOVA analyses. The 2-way ANOVA analyses with emotion as a withinsubject factor and group as a between-subject factor showed that the 2-way interaction effect in the implicit task was not significant $\left(F_{1,36}=0.14, P>0.5\right)$, while notably it was significant in the explicit task $\left(F_{1,36}=5.25, P<0.05\right)$. We, in turn, explored the group effect in negative and neutral conditions, respectively, with two separate $t$-tests. The results revealed that GAD patients showed a significantly shorter reaction time than the control group under the negative condition during the explicit task $\left(t_{36}=2.21, P<0.05\right)$. The difference between the two groups under neutral $\left(t_{36}=0.49, P>0.5\right)$ conditions was not significant.

\section{ERP Data Analysis \\ P1}

The 5-way ANOVA analyses with task, emotion, trial type, and electrode as within-subject factors and group as the betweensubject factor did not reveal any significant main effect or interaction effect in P1 amplitudes. P1 latency showed a significant main effect at task $\left(F_{1,36}=5.15, P<0.05\right)$ and group $\left(F_{1,36}=6.16\right.$, $P<0.05)$. A shorter $P 1$ latency was elicited in the implicit task (115.89 ms) compared to the explicit task (119.58 ms). The anxiety group $(112.68 \mathrm{~ms})$ showed a shorter P1 latency than the control group (122.79 ms). As P1 latency showed task and group main effects, we present the $\mathrm{P} 1$ result average across task, emotion and group in Figure 2A.

\section{N170}

N170 amplitudes showed a significant main effect for task $\left(F_{1,36}=24.36, P<0.001\right)$. The faces in the explicit task $(-2.98 \mu \mathrm{V})$ elicited larger N170 amplitudes than that in the implicit task $(-2.12 \mu \mathrm{V})$. The group $\times$ electrodes interaction effect showed that the anxiety group $(-4.14 \mu \mathrm{V}$; $4.09 \mu \mathrm{V})$ showed larger amplitudes than the control group $(-1.92 \mu \mathrm{V} ;-1.16 \mu \mathrm{V})$ at P8 and PO8 electrode sites, respectively $\left(F_{1,36}=6.24, P<0.05\right.$; Figure 2B $)$. The amplitude differences between the two groups at P7 and PO7 electrode sites were not significant $\left(F_{1,36}=0.14, P>0.7\right)$. The N170 latency showed a significant group $\left(F_{1,36}=4.96\right.$, 
TABLE 2 | Amplitudes in P, N170, VPP, N2, P3 segment of the study participants.

\begin{tabular}{|c|c|c|c|c|c|c|c|c|}
\hline & \multicolumn{4}{|c|}{ Explicit task } & \multicolumn{4}{|c|}{ Implicit task } \\
\hline & \multicolumn{2}{|c|}{ Negative } & \multicolumn{2}{|c|}{ Neutral } & \multicolumn{2}{|c|}{ Negative } & \multicolumn{2}{|c|}{ Neutral } \\
\hline & Go & No-go & Go & No-go & Go & No-go & Go & No-go \\
\hline P1 (GAD) & $5.0(0.7)$ & $5.6(0.7)$ & $5.3(0.7)$ & $5.4(0.7)$ & $5.0(0.7)$ & $5.4(0.7)$ & $5.0(0.7)$ & $5.4(0.7)$ \\
\hline P1 (CON) & $5.5(0.7)$ & $5.8(0.7)$ & $5.5(0.7)$ & $5.8(0.7)$ & $5.1(0.7)$ & $5.5(0.8)$ & $5.1(0.7)$ & $5.8(0.7)$ \\
\hline N170 (GAD) & $-2.8(0.6)$ & $-2.7(0.6)$ & $-2.8(0.6)$ & $-2.6(0.6)$ & $-3.8(0.6)$ & $-3.8(0.6)$ & $-3.9(0.5)$ & $-3.8(0.6)$ \\
\hline N170 (CON) & $-1.1(0.6)$ & $-1.5(0.6)$ & $-1.8(0.6)$ & $-1.4(0.6)$ & $-2.1(0.6)$ & $-2.4(0.6)$ & $-2.1(0.5)$ & $-2.0(0.6)$ \\
\hline VPP (GAD) & $9.0(0.8)$ & $9.4(0.9)$ & $8.3(0.8)$ & $9.0(0.8)$ & $8.3(0.8)$ & $8.4(0.9)$ & $7.9(0.8)$ & $8.0(0.8)$ \\
\hline VPP (CON) & $6.9(0.8)$ & $7.2(0.8)$ & $6.0(0.8)$ & $6.1(0.8)$ & $6.0(0.8)$ & $6.1(0.9)$ & $5.8(0.8)$ & $5.6(0.9)$ \\
\hline N2 (GAD) & $4.4(0.7)$ & $4.2(0.8)$ & $3.8(0.8)$ & $4.3(0.7)$ & $2.7(0.7)$ & $2.3(0.8)$ & $2.6(0.7)$ & $2.1(0.8)$ \\
\hline N2 (CON) & $3.9(0.7)$ & $3.4(0.8)$ & $2.7(0.8)$ & $2.6(0.7)$ & $1.8(0.7)$ & $1.7(0.8)$ & $1.8(0.7)$ & $1.5(0.8)$ \\
\hline P3 (GAD) & $5.8(0.7)$ & $7.9(0.9)$ & $6.1(0.7)$ & $6.5(0.8)$ & $4.6(0.7)$ & $5.8(0.9)$ & $4.8(0.8)$ & $6.0(0.9)$ \\
\hline P3 (CON) & $7.0(0.7)$ & $8.7(0.9)$ & $5.5(0.7)$ & $6.8(0.8)$ & $4.0(0.7)$ & $6.4(0.9)$ & $4.0(0.8)$ & $6.6(0.9)$ \\
\hline
\end{tabular}

Amplitudes in microvolt. All values expressed in mean (SD). GAD, general anxiety disorder; CON, control.

$P<0.05)$ main effect. The anxiety group $(159.48 \mathrm{~ms})$ showed a shorter N170 latency than the control group (168.51 ms).

\section{VPP}

Task, emotion, and electrodes had an effect on the VPP amplitude $\left(F_{1,36}=14.11, P=0.001 ; F_{1,36}=30.13, P<0.001 ; P<0.001\right.$; $F_{1,36}=3.21, P<0.05$; The VPP amplitude was larger in the explicit task $(7.78 \mu \mathrm{V})$ than in the implicit task $(7.03 \mu \mathrm{V})$. Sad faces $(7.69 \mu \mathrm{V})$ elicited a larger amplitude than neutral faces $(7.12 \mu \mathrm{V})$. The amplitudes of FCz $(8.06 \mu \mathrm{V}, P<0.05)$ and $\mathrm{Cz}$ $(8.29 \mu \mathrm{V}, P<0.05)$ were significantly larger than the other sites. Moreover, we detected that the group main effect was marginally significant $\left(F_{1,36}=3.85, P=0.057\right)$. The VPP amplitude in the anxiety group $(8.58 \mu \mathrm{V})$ was somewhat larger than that in the control group $(6.23 \mu \mathrm{V}$; Figure $2 \mathrm{C})$.

The main effects of emotion and electrodes in VPP latency were significant $\left(F_{1,36}=11.90, P=0.001 ; F_{1,36}=3.89, P<0.05\right)$. The VPP latency elicited by sad faces $(182.96 \mathrm{~ms})$ was shorter than that elicited by neutral faces $(186.77 \mathrm{~ms})$. The latency of $\mathrm{FCz}(180.33 \mathrm{~ms}, P<0.05)$ and $\mathrm{Cz}(180.15 \mathrm{~ms}, P<0.05)$ was significantly shorter than the other sites.

\section{N2}

The N2 amplitudes showed a significant main effect for task, emotion, and electrodes $\left(F_{1,36}=42.91, P<0.001 ; F_{1,36}=12.35\right.$, $P=0.001 ; F_{8,288}=23.2$ respectively, $\left.P<0.001\right)$. The explicit task $(3.71 \mu \mathrm{V})$ elicited larger amplitudes than the implicit task $(2.11 \mu \mathrm{V})$. The amplitude elicited by sad faces $(3.09 \mu \mathrm{V})$ was larger than that elicited by neutral faces $(2.73 \mu \mathrm{V})$. The N2 amplitudes were larger at FC3 $(1.54 \mu \mathrm{V}, P<0.05), \mathrm{FCz}(0.56 \mu \mathrm{V}$, $P<0.001)$, and FC4 $(1.82 \mu \mathrm{V}, P<0.05)$ than the other sites. We detected that the trial type and electrode interaction effect was significant $\left(F_{1,36}=3.97, P<0.05\right)$. The simple analysis showed that the amplitudes elicited by no-go trials $(1.02 \mu \mathrm{V})$ were larger than go trials $(1.36 \mu \mathrm{V})$ at FCz and FC4 $\left(F_{8,288}=4.66, P<0.05\right)$. The trial type effect was not significant at the other sites. More importantly, the task, trial type, electrode, and group interaction effect were significant $\left(F_{8,288}=2.66, P<0.05\right.$; Figure 3). To clearly illustrate this interaction effect, we calculated the no-go/go difference waves to observe the dissimilarity between the anxiety and control groups.

The main effects of trial type in N2 latency were significant $\left(F_{1,36}=4.62, P<0.05\right)$. The N2 latency elicited by go stimuli $(300.74 \mathrm{~ms})$ was shorter than that elicited by no-go stimuli (309.07 ms).

\section{No-go/go Differences in Event-Related Potentials at the N2 Interval}

No-go/go differences in event-related potentials were presented in Figure 4. The interaction effect between task, trial type, electrode, and group was significant on the amplitudes at the N2 interval. To expound the features of this interaction, we focused analysis on the difference wave of no-go minus go conditions. The 4-way multivariate ANOVA on the N2 amplitudes revealed significant task, electrode, and group interaction effects $\left(F_{8,288}=2.66, P<0.05\right)$. The simple analysis revealed that the control group $(-0.71 \mu \mathrm{V})$ showed larger amplitudes than the anxiety group $(0.08 \mu \mathrm{V})$ in explicit tasks at the right fronto-central sites $\left(F_{1,36}=3.47, P=0.07\right)$. The amplitudes showed a larger tendency in the GAD group $(-0.7 \mu \mathrm{V})$ than in the control group $(-0.2 \mu \mathrm{V})$, but the difference was not significant in the implicit task $\left(F_{1,36}=1.81, P>0.1\right)$.

\section{P3}

Because the time window was long, P3 was divided into two segmentations, 450-550 ms and 550-650 ms. During the 450$550 \mathrm{~ms}$ time window (Figure 5), P3 amplitudes showed a significant main effect for task, emotion, trial type, and electrode $\left(F_{1,36}=28.06, P<0.001 ; F_{1,36}=13.29, P=0.001 ; F_{1,36}=24.62\right.$, $P<0.001 ; F_{8,288}=12.61$, respectively, $\left.P<0.001\right)$. The amplitudes were larger during the explicit task $(6.80 \mu \mathrm{V})$ than in the implicit task (5.33). Sad faces $(6.32 \mu \mathrm{V})$ elicited larger amplitudes than neutral faces $(5.81 \mu \mathrm{V})$. No-go trials $(6.87 \mu \mathrm{V})$ elicited larger amplitudes than go trials $(5.26 \mu \mathrm{V}) . \mathrm{Pz}(8.19 \mu \mathrm{V})$ elicited the largest amplitudes of P3. $\mathrm{Cz}(6.84 \mu \mathrm{V}), \mathrm{C} 4(6.74 \mu \mathrm{V})$, and 


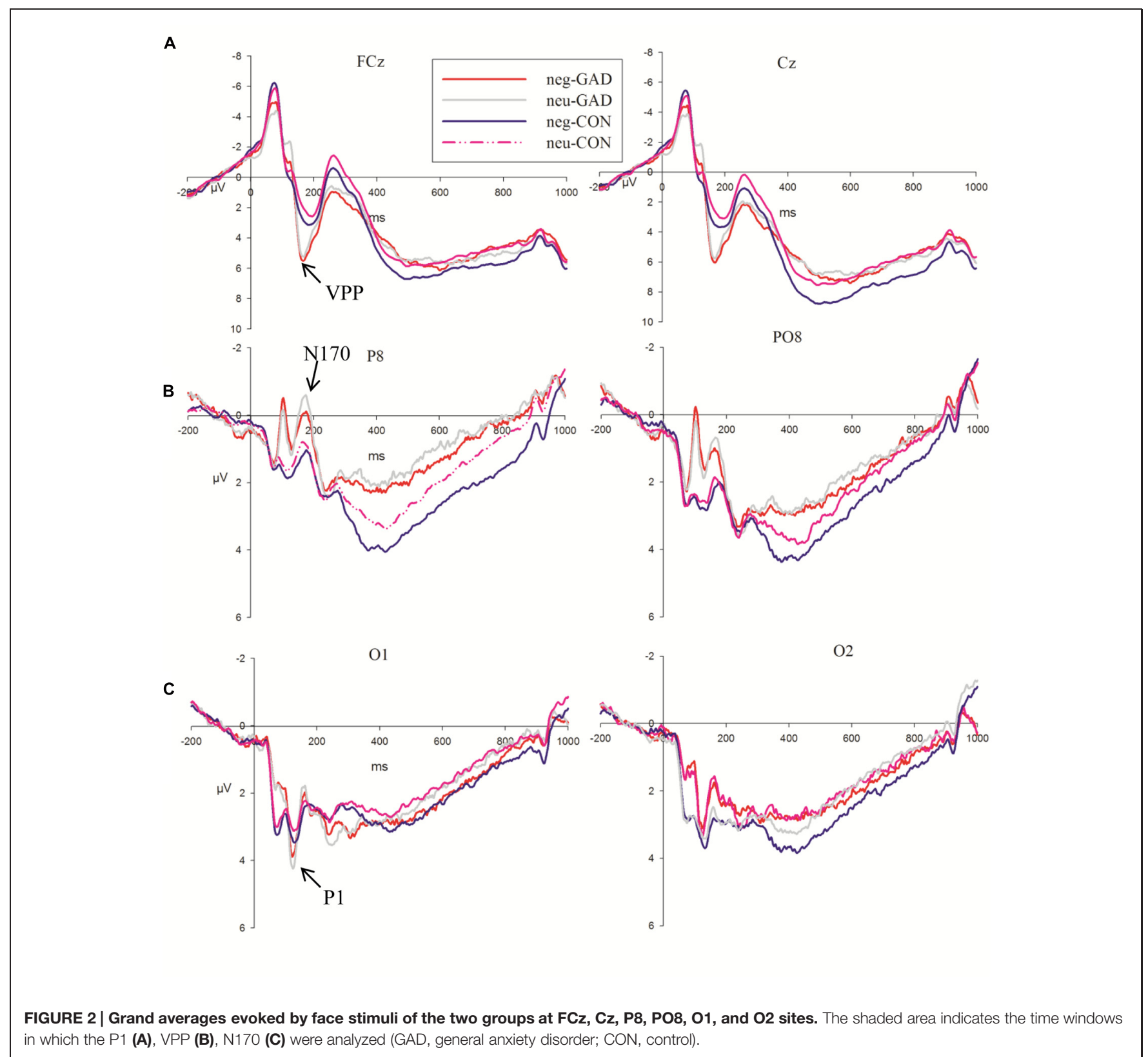

P4 $(6.29 \mu \mathrm{V})$ electrodes elicited larger amplitudes than the other sites. We found that the interaction effect between task, emotion, and trial type was marginally significant $\left(F_{1,36}=3.74, P=0.06\right.$; Figure 5). The simple analysis revealed that no-go/P3 amplitudes elicited by sad faces $(8.31 \mu \mathrm{V})$ were larger than those elicited by neutral faces $(6.67 \mu \mathrm{V})$ in the explicit task. The no-go amplitude differences between sad $(6.18 \mu \mathrm{V})$ and neutral $(6.35 \mu \mathrm{V})$ conditions were not significant in the implicit task.

During the 550-650 ms time window, P3 amplitudes showed significant task, emotion, trial type, and electrode main effects $\left(F_{1,36}=29.55, P<0.001 ; F_{1,36}=7.32, P=0.01 ; F_{1,36}=41.64\right.$, $P<0.001 ; F_{8,288}=13.93$, respectively, $\left.P<0.001\right)$. P3 amplitudes were larger in the explicit task $(6.98 \mu \mathrm{V})$ than in the implicit task $(5.61 \mu \mathrm{V})$. Sad faces $(6.51 \mu \mathrm{V})$ elicited larger amplitudes than neutral faces $(6.07 \mu \mathrm{V})$. No-go trials $(7.21 \mu \mathrm{V})$ elicited larger amplitudes than in go trials $(5.38 \mu \mathrm{V})$. The amplitudes at $\mathrm{Cz}$ $(7.62 \mu \mathrm{V})$ and $\mathrm{Pz}(7.74 \mu \mathrm{V})$ were larger than the other sites.

The main effects of trial type in P3 latency were significant $\left(F_{1,36}=31.95, P<0.001\right)$. The P3 latency was longest at $\mathrm{Cz}$ (611.98 ms).

\section{Relationship between Clinical Characteristics and Task-Related Measures}

Significant correlations were not found between the clinical measures (HAMA) and the discrimination accuracy, which also occurred in the relationship of clinical measures and no-go/N2 amplitudes in the explicit task. We did not find correlations between no-go/N2 and behavior accuracy. 


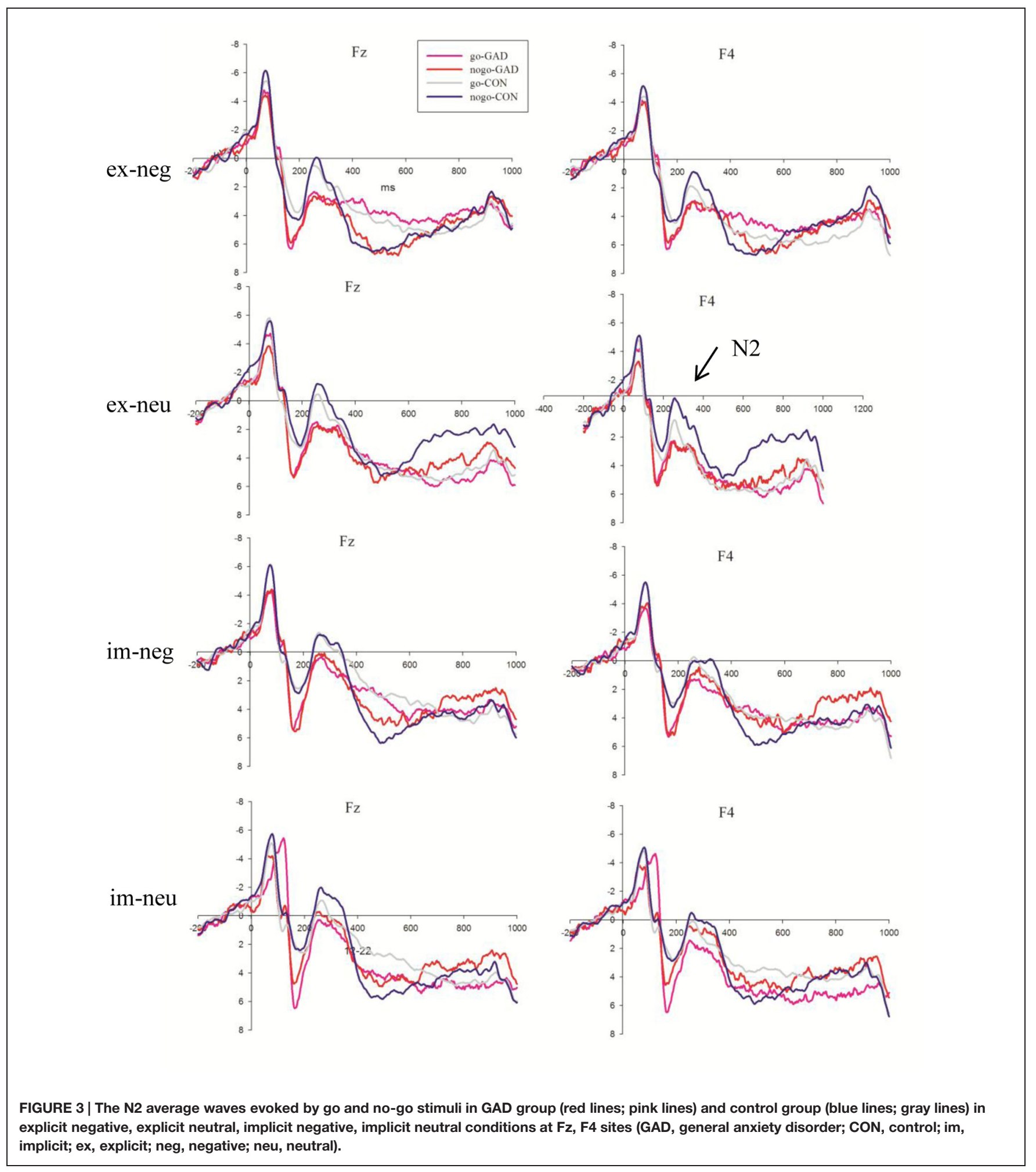

\section{Source-Localization Data}

The voxel-based whole-brain sLORETA images for control and anxiety groups under no-go conditions at the N2 interval were compared using non-parametric randomization tests in order to identify the cortical regions involved. As hypothesized, the brain regions involved in response inhibition processing varied between the control and anxiety groups in the explicit $\left(t_{36}=1.29\right.$, $P<0.05)$ and implicit tasks $\left(t_{36}=1.29, P<0.05\right)$. The current source density (CSD) in the right DLPFC (Brodmann 9, $\max$ values obtained at $x=50, y=20$, and $z=40$ ) 

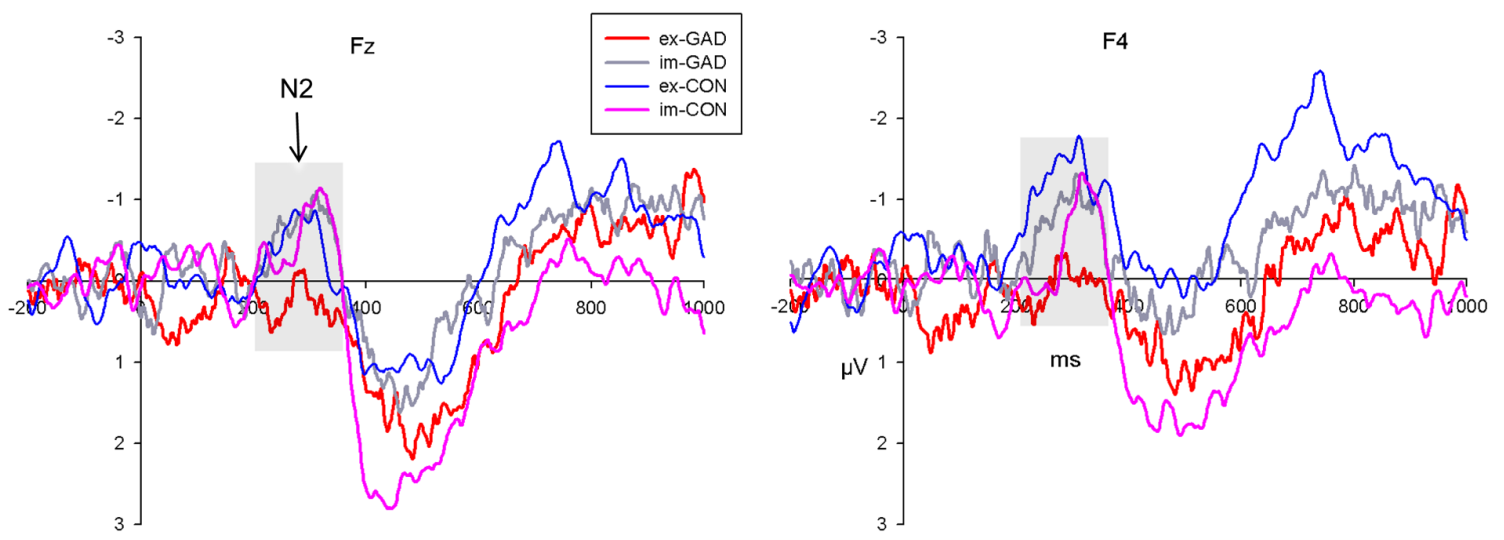

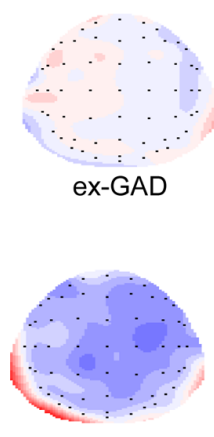

im-GAD
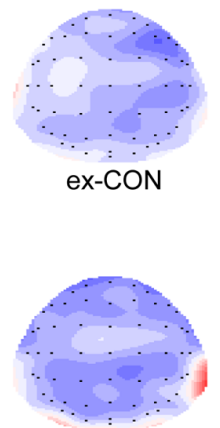

im-CON
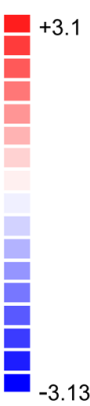

$-3.13$

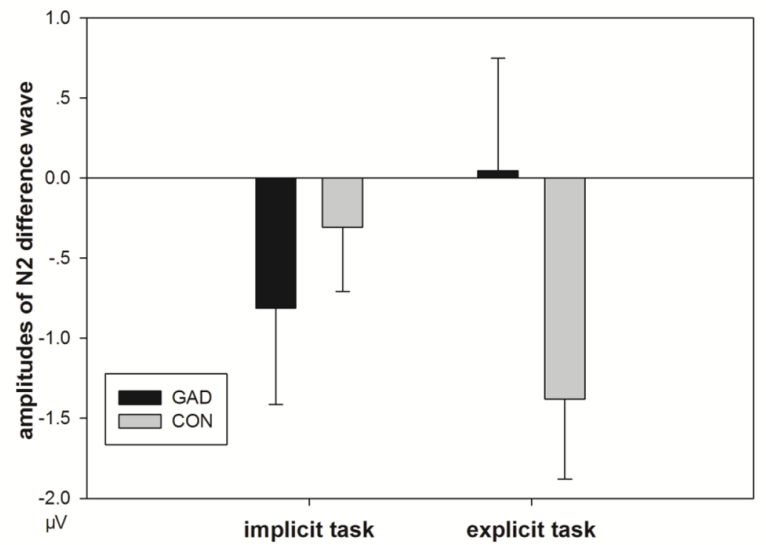

FIGURE 4 | The N2 difference waves of No-go minus Go trials at Fz, F4 sites (upper) and corresponding amplitudes histogram (bottom right) and scalp topographies (bottom left) of the two groups during implicit and explicit tasks (GAD, general anxiety disorder; CON, control; im, implicit; ex, explicit).
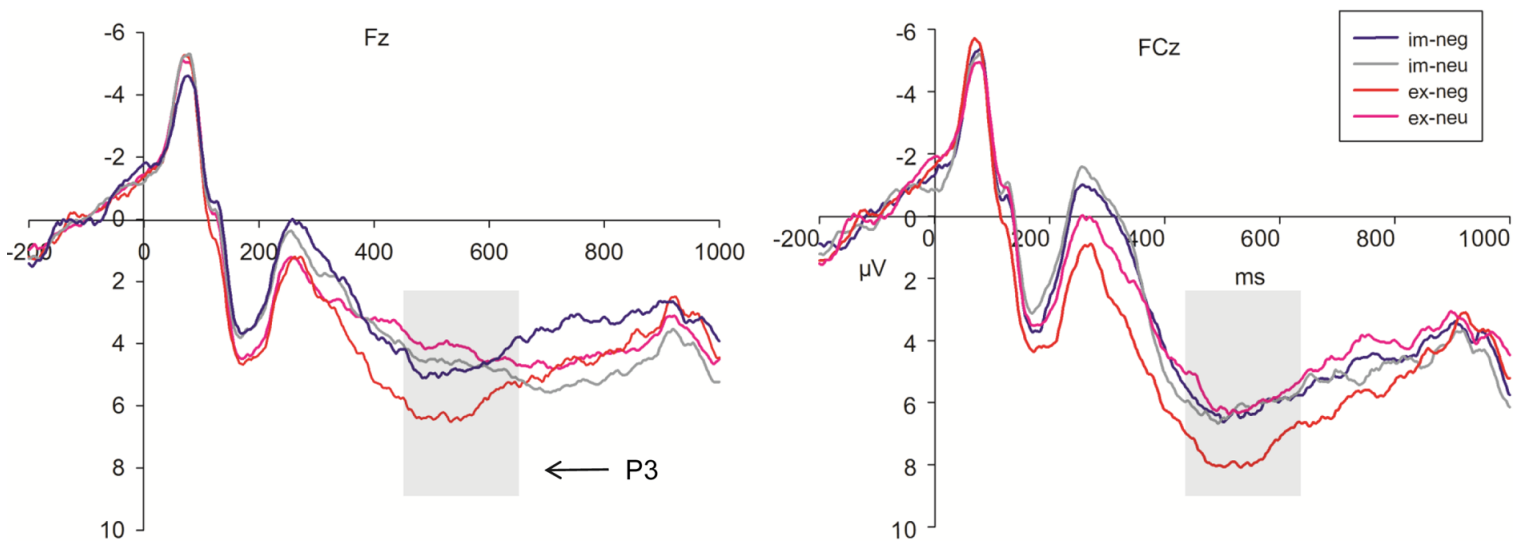

FIGURE 5 | Grand averages evoked by negative and neutral stimuli under implicit task (blue lines; gray lines) and explicit task (red lines; pink lines) in No-go trials at Fz, FCz sites (im, implicit; ex, explicit; neg, negative; neu, neutral).

in the control group was larger than in the anxiety group. Moreover, the current source density in the left superior temporal gyrus (ISTG, Brodmann 42, max values obtained at $x=-60$, $y=-30$, and $z=15$ ) and left inferior parietal Lobule (IIPL,
Brodmann 40, max values obtained at $x=-60, y=-45$, and $z=20$ ) were larger in the anxiety group than in the control group in the implicit task $\left(t_{36}=-1.21, P<0.05\right.$; Figure 6). 

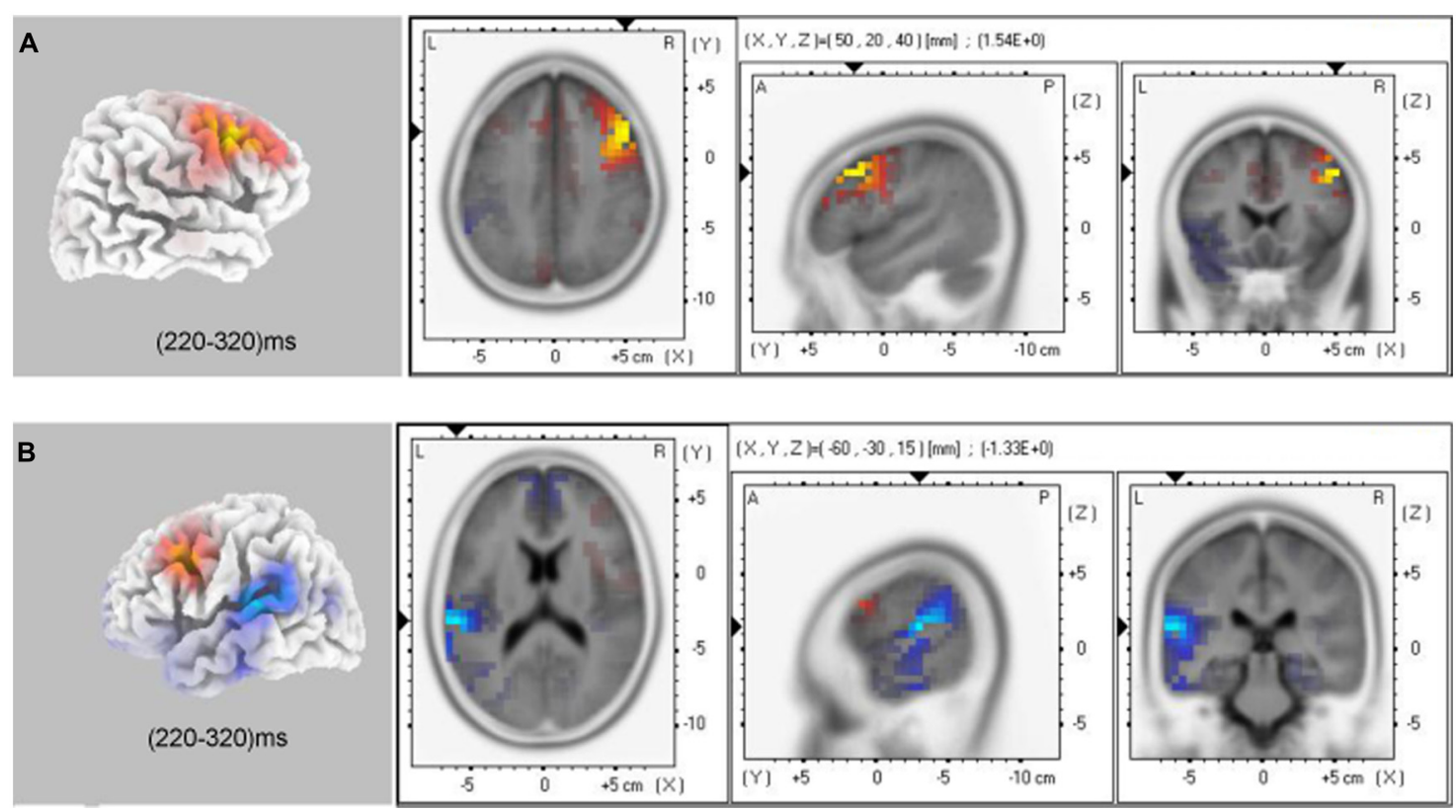

FIGURE 6 | sLORETA solutions to non-parametric randomization tests on N2 components showing voxels in which the CON group > GAD group contrast (A) and GAD group $>$ CON group contrast $(B)$ were significant $(P<0.05$; GAD, general anxiety disorder; CON, control).

\section{Discussion}

The present study aimed to examine the neural substrates of response inhibition to sad faces across implicit and explicit tasks in clinical GAD individuals using high time resolution ERP methods. The GAD group showed smaller amplitudes of no-go/go difference waves across sad and neutral emotions specifically in the explicit task rather than in the implicit task at the $\mathrm{N} 2$ interval when compared to controls. The source localization of no-go/N2 components showed a lower CSD of rDLPFC in both implicit and explicit tasks and a higher CSD of ISTG and IIPL in implicit tasks in GAD individuals compared to the control group. Thus, our results provide further evidence for the dissociation at a neural level of response inhibition to sad faces between implicit and explicit tasks.

As shown in Figure 2, at the $60-140 \mathrm{~ms}$ interval, a posterior P1 component reflecting early visual processing was induced by facial stimuli. As expected, the present study observed that the GAD group showed a shorter latency than the control group, irrespective of task, and emotional conditions. In addition, the faces in the present study induced prominent face-specific N170 and VPP components. The GAD group showed larger N170 and VPP amplitudes relative to the control group. This result was consistent with previous studies, which reported that $\mathrm{N} 170$ and VPP can be modulated by attention (Holmes et al., 2003; Mohamed et al., 2009). As usual, GAD patients were sensitive to facial stimuli and they allocated more attention resources to face processing relative to the control group (Douilliez et al., 2012; Peschard et al., 2013). The result was similar to previous studies reporting that anxious individuals exhibit a faster attention capture to facial expression and evince faster latency in early ERP components (Russell, 1989; Peschard et al., 2013). The above results suggest that the GAD group automatically shows an attention bias to face processing at an early stage.

More remarkable, this study observed a significant interaction effect between the task and group for the no-go/go difference wave at the $220-320 \mathrm{~ms}$ interval. As indicated in Figure 4, the subtraction of ERPs elicited by go stimuli from those elicited by no-go stimuli generated a clear no-go related N2 component during this stage. The amplitudes of no-go related N2 were significantly smaller in the GAD group compared to the control group in the explicit task, irrespective of the emotion type. Previous ERP studies also found that anxious children showed decreased no-go/go difference wave amplitudes during the N2 stage (Hum et al., 2013) and nogo/N2 was negatively related to anxiety-related personality traits (Sehlmeyer et al., 2010). The present study further found that GAD patients showed decreased no-go/go difference waves at the N2 interval in the explicit task compared to the control group. As previously mentioned, the no-go related N2 amplitude was accepted as the index of conflict monitoring and for the increased attention engagement that forms the basis for the subsequent process of inhibition (Yuan et al., 2008). In explicit task, facial expression is within the voluntary attention scope and is directly processed. In implicit task facial expression is within the involuntary attention scope, and therefore is incidentally processed. Thus, the attention resource for emotion processing is distinct between the two conditions. The difference of N2 amplitudes between GAD and control was dissociated between the two tasks. These results suggested that response inhibition of GAD individuals were modulated by attention at the conflict monitoring stage. From these results, we 
concluded that the processing of response inhibition was interrupted in GAD when subjects were asked to respond to facial expressions. In this situation, GAD patients were more sensitive to the directly processed facial expressions and devoted more attention resources to facial expression processing. According to the shared resources theory, emotional processing requires cognitive resources and consumes the common pool of such resources with top-down cognitive control (Pessoa, 2009). Thus, the current direct goal of response inhibition was interfered by increased response-competition of the task-irrelevant distracters (Berggren and Derakshan, 2013). These results were consistent with previous studies that indicated that anxiety shows a response inhibition deficit using emotional distracters (Cisler and Koster, 2010; Berggren and Derakshan, 2013). In addition, GAD also showed decreased amplitudes of $\mathrm{N} 2$ difference wave in neutral condition in explicit task. Previous study have suggested that social anxiety disorder patients tend to interpret neutral and other ambiguous emotional stimuli negatively (Winton et al., 1995). Neuroimaging studies found that amygdala showed a different activation pattern in response to neutral faces compared to controls (Cooney et al., 2006; Veit et al., 2006). In the present study, GAD patients may also interpret neutral faces negatively and showed abnormal response inhibition to neutral faces.

In contrast to the explicit task, the implicit task showed somewhat larger N2 amplitudes in the GAD group compared to the control group even though the difference was not significant. In the implicit task, participants were introduced to responses or suppressed their responses according to face gender. The attention was diverted from facial expressions. Gross has proposed that the emotional effect on cognition was weakened when attention was disengaged from the emotional aspects of stimuli (Gross, 2001; Ochsner and Gross, 2005). Consistent with Gross' work, the present results indicated that response inhibition to social negative stimuli in GAD individuals could be regulated by attention resources. When introduced to disengage attention from negative events to nonemotional aspects, GAD individuals showed an improved ability to inhibit responses to social information. These results support attention bias modification treatment therapy in GAD individuals. The attention bias modification means training attention to tend to positive or neutral stimulus and to avoid negative stimulus. A large number of studies reported that after attention bias modification treatment, the anxiety symptom in GAD decreased significantly (Browning et al., 2010; Hakamata et al., 2010; Hallion and Ruscio, 2011; MacLeod and Mathews, 2012). The present study further provides neural evidence that the cognitive processing was modified in GAD when asked to divert attention from emotional to non-emotional aspects. However, the present results were inconsistent with previous studies that reported that facial processing was not affected by task instructions in social anxiety individuals. The participants showed attention bias to faces in both implicit and explicit tasks (Peschard et al., 2013). This may be because the previous study only investigated the modulated effect of task instruction on face processing but not the effect of the emotion on response inhibition, as in the present study. Another reason was that the participants in that study had subclinical social anxiety and the present study included general anxiety individuals. There could be differences in neural mechanisms of interaction of emotion and cognition between subclinical social anxiety and GAD.

By using signal detection theory, we found that GAD showed poor discretionary accuracy of $d^{\prime}$ compared to controls when asked to respond to sad faces and inhibit motor responses to neutral faces in the explicit task. This was interpreted as emotional faces inducing an amplified vigilance in GAD. Thus, when asked to response to the sad faces, they made a faster response to the stimuli and the error rate increased. The results were consistent with previous studies, which also found anxiety individuals showed faster responses to threatening and happy faces (Bradley et al., 1999; Rossignol et al., 2005). Several studies reported that anxiety individuals showed attention bias to social threatening stimuli (fearful or angry faces; Bradley et al., 1999; Fox, 2002; Pergamin-Hight et al., 2014). The present study further found that GAD patients also showed attention bias to sad faces when processed explicitly. In addition, we calculated the correlation between discrimination accuracy and clinical characteristics and response times and clinical characteristics. However, the correlation was not significant. The results may indicate that attention bias to sad faces in the explicit task is an endophenotype of GAD and unrelated to the clinical characteristics. While in the implicit task, there were no significant differences in behavioral performances between GAD patients and the control group. Previous studies have revealed that, although anxious individuals showed reduced response efficiency (i.e., the pattern in which cognitive resources are utilized to achieve the desirable performance outcome), the performance effectiveness (i.e., the ability to perform the task) was well preserved relative to controls (Righi et al., 2009; Ansari and Derakshan, 2011; Berggren and Derakshan, 2013). The reduced cognitive efficiency may be ameliorated by strategies such as a compensatory effort (Eysenck et al., 2007). However, other studies reported that anxious individuals showed impaired performance effectiveness because of interruption by task-irrelevant sad faces (Bishop, 2009; Ansari and Derakshan, 2011; Basten et al., 2011). The present study found that in the implicit task there was no difference in discrimination accuracy and response time. Performance effectiveness in the explicit task was decreased in the GAD group. According to the shared resources theory, the processing of facial expressions occupied even more cognitive resources and the remaining resources for compensatory efforts on the current direct goal were limited (Pessoa, 2009). Thus, the present results reconcile the conflict by showing that that the performance effectiveness was well preserved in implicit task, but not in explicit task.

After a conflict monitoring process indicated by N2, an inappropriate response was inhibited at the P3 stage (Bokura et al., 2001; Albert et al., 2010). Similar to the results of our previous study (Yu et al., 2014), we observed a task, emotion, and trial type interaction effect at the P3 interval (see details in Figure 5). In the explicit task, inhibiting negative faces elicited larger P3 amplitudes than neutral faces. However, the difference in nogo/P3 amplitudes between sad and neutral conditions was not significant during the implicit task. In this task, participants 
were instructed to respond to or inhibit their response according to facial gender, so facial expressions were task-irrelevant stimuli and their attentions were diverted from facial expressions. The intensity of negative emotion was decreased when the attention was disengaged from the emotional stimuli in the implicit task compared to the explicit task (Pessoa et al., 2005; Schonfelder et al., 2013), thus resulting in a reduced emotional effect on response inhibition.

Using sLORETA source localization analysis during the nogo/N2 time window, we observed that the GAD group displayed decreased CSD of rDLPFC compared to the control group across implicit and explicit tasks (Figure 6). Neuroimaging research in humans identified that the rDLPFC, as part of the rIFC, plays a critical role in response inhibition, task switching, updating, and other cognitive control functions (Verbruggen et al., 2010; Meyer et al., 2011). Convergent reports have emphasized the key role of DLPFC in the pathophysiology of GAD individuals (Meyer et al., 2011). Consistently, the present results further revealed a decreased ability of inhibition to task-irrelevant items and the dysfunction of rDLPFC may be an important cognitive and biological mechanism of GAD individuals. In addition, we observed that, in the implicit task, GAD individuals showed a significantly higher CSD in the junction of ISTG and IIPL (BA 42/40) relative to the control group. Previous neuroimaging researches reported that STG and IPL were part of a network for voluntary attention control and were involved in the processing of attention selection, attention shifting, and working memory in the general population (Hopfinger et al., 2000; Shapiro et al., 2002; Achiron et al., 2013). The abnormal structure and function in these areas led to impaired cognitive performance (Behrmann et al., 2004; Achiron et al., 2013). In the present study, the ISTG and IPLbrain regions were involved in allocating more attention resources to perform the implicit tasks and compensate for the function of rDLPFC in GAD individuals. However, the source localization method was based on the

\section{References}

Aarts, K., and Pourtois, G. (2010). Anxiety not only increases, but also alters early error-monitoring functions. Cogn. Affect. Behav. Neurosci. 10, 479-492. doi: 10.3758/CABN.10.4.479

Achiron, A., Chapman, J., Tal, S., Bercovich, E., Gil, H., and Achiron, A. (2013). Superior temporal gyrus thickness correlates with cognitive performance in multiple sclerosis. Brain Struct. Funct. 218, 943-950. doi: 10.1007/s00429-0120440-3

Albert, J., Lopez-Martin, S., and Carretie, L. (2010). Emotional context modulates response inhibition: neural and behavioral data. Neuroimage 49, 914-921. doi: 10.1016/j.neuroimage.2009.08.045

Ansari, T. L., and Derakshan, N. (2011). The neural correlates of cognitive effort in anxiety: effects on processing efficiency. Biol. Psychol. 86, 337-348. doi: 10.1016/j.biopsycho.2010.12.013

Basten, U., Stelzel, C., and Fiebach, C. J. (2011). Trait anxiety modulates the neural efficiency of inhibitory control. J. Cogn. Neurosci. 23, 3132-3145. doi: 10.1162/jocn_a_00003

Behrmann, M., Geng, J. J., and Shomstein, S. (2004). Parietal cortex and attention. Curr. Opin. Neurobiol. 14, 212-217. doi: 10.1016/j.conb.2004. 03.012

Berggren, N., and Derakshan, N. (2013). Attentional control deficits in trait anxiety: why you see them and why you don't. Biol. Psychol. 92, 440-446. doi: 10.1016/j.biopsycho.2012.03.007 proposition from a mathematical, and not a physiological, standpoint. Future research employing a wide range of experimental tasks and designs, as well as brain imaging methodologies that may improve the spatial resolution, such as fMRI, is needed to substantiate and extend these findings.

The present study has several limitations. First, we only used the sad expressions as experimental stimuli and how other basic emotions interact in response inhibition in GAD across implicit and explicit tasks warrants investigation. Second, the dissociation of electrophysiological and behavioral difference between implicit and explicit tasks may due to the difficulty difference. To investigate the difficulty difference between the two tasks, we used signal detection theory to analyze the behavior performance. The discrimination accuracy and decision bias did not show differences between the implicit and explicit tasks in both groups. However, the statistical methods could not analyze this objective error well. We will manipulate the difficulty of the two tasks as a variable in later experiments.

\section{Conclusion}

The present results demonstrated that GAD individuals show deficits when inhibiting responses to sad faces in an explicit task rather than in an implicit task. The amplitudes of no-go/go difference waves at the $\mathrm{N} 2$ interval were significantly smaller in GAD individuals than in controls during the explicit task, but showed a reversed trend during the implicit task. In addition, the results confirmed that GAD participants displayed a smaller rDLPFC in the no-go condition, which indicated that dysfunction of rDLPFC may play a critical role in the biological mechanism of GAD. The larger ISTG/1PL activation in GAD individuals in the implicit task may compensate for the dysfunction of rDLPFC. These results provided evidence for attention bias modification treatment in GAD individuals.

Berkman, E. T., Burklund, L., and Lieberman, M. D. (2009). Inhibitory spillover: intentional motor inhibition produces incidental limbic inhibition via right inferior frontal cortex. Neuroimage 47, 705-712. doi: 10.1016/j.neuroimage.2009.04.084

Bishop, S. J. (2009). Trait anxiety and impoverished prefrontal control of attention. Nat. Neurosci. 12, 92-98. doi: 10.1038/nn.2242

Bokura, H., Yamaguchi, S., and Kobayashi, S. (2001). Electrophysiological correlates for response inhibition in a Go/NoGo task. Clin. Neurophysiol. 112, 2224-2232. doi: 10.1016/S1388-2457(01)00691-5

Botvinick, M. M., Braver, T. S., Barch, D. M., Carter, C. S., and Cohen, J. D. (2001). Conflict monitoring and cognitive control. Psychol. Rev 108, 624-652. doi: 10.1037/0033-295X.108.3.624

Bradley, B. P., Mogg, K., White, J., Groom, C., and de Bono, J. (1999). Attentional bias for emotional faces in generalized anxiety disorder. Br. J. Clin. Psychol. 38(Pt 3), 267-278. doi: 10.1348/014466599162845

Browning, M., Holmes, E. A., and Harmer, C. J. (2010). The modification of attentional bias to emotional information: a review of the techniques, mechanisms, and relevance to emotional disorders. Cogn. Affect. Behav. Neurosci. 10, 8-20. doi: 10.3758/CABN.10.1.8

Cisler, J. M., and Koster, E. H. (2010). Mechanisms of attentional biases towards threat in anxiety disorders: an integrative review. Clin. Psychol. Rev. 30, $203-$ 216. doi: 10.1016/j.cpr.2009.11.003

Cooney, R. E., Atlas, L. Y., Joormann, J., Eugene, F., and Gotlib, I. H. (2006). Amygdala activation in the processing of neutral faces in social 
anxiety disorder: is neutral really neutral? Psychiatry Res. 148, 55-59. doi: 10.1016/j.pscychresns.2006.05.003

Decker, M. L., Turk, C. L., Hess, B., and Murray, C. E. (2008). Emotion regulation among individuals classified with and without generalized anxiety disorder. $J$. Anxiety Disord. 22, 485-494. doi: 10.1016/j.janxdis.2007.04.002

Dierks, T., Jelic, V., Pascual-Marqui, R. D., Wahlund, L., Julin, P., Linden, D. E., et al. (2000). Spatial pattern of cerebral glucose metabolism (PET) correlates with localization of intracerebral EEG-generators in Alzheimer's disease. Clin. Neurophysiol. 111, 1817-1824. doi: 10.1016/S1388-2457(00)00427-2

Donkers, F. C., and van Boxtel, G. J. (2004). The N2 in go/no-go tasks reflects conflict monitoring not response inhibition. Brain Cogn. 56, 165-176. doi: 10.1016/j.bandc.2004.04.005

Douilliez, C., Yzerbyt, V., Gilboa-Schechtman, E., and Philippot, P. (2012). Social anxiety biases the evaluation of facial displays: evidence from single face and multi-facial stimuli. Cogn. Emot. 26, 1107-1115. doi: 10.1080/02699931.2011.632494

Duerden, E. G., Taylor, M. J., Soorya, L. V., Wang, T., Fan, J., and Anagnostou, E. (2013). Neural correlates of inhibition of socially relevant stimuli in adults with autism spectrum disorder. Brain Res. 1533, 80-90. doi: 10.1016/j.brainres.2013.08.021

Dziobek, I., Preissler, S., Grozdanovic, Z., Heuser, I., Heekeren, H. R., and Roepke, S. (2011). Neuronal correlates of altered empathy and social cognition in borderline personality disorder. Neuroimage 57, 539-548. doi: 10.1016/j.neuroimage.2011.05.005

Eysenck, M. W., Derakshan, N., Santos, R., and Calvo, M. G. (2007). Anxiety and cognitive performance: attentional control theory. Emotion 7, 336-353. doi: 10.1037/1528-3542.7.2.336

Fabiani, M., Gratton, G., and Goles, M. G. (2000). Hand Book of Psychophysiology. Cambridge University Press: London.

Fox, E. (2002). Processing emotional facial expressions: the role of anxiety and awareness. Cogn. Affect. Behav. Neurosci. 2, 52-63. doi: 10.3758/CABN.2.1.52

Frenkel, T. I., and Bar-Haim, Y. (2011). Neural activation during the processing of ambiguous fearful facial expressions: an ERP study in anxious and nonanxious individuals. Biol. Psychol. 88, 188-195. doi: 10.1016/j.biopsycho.2011.08.001

Gehricke, J., and Shapiro, D. (2000). Reduced facial expression and social context in major depression: discrepancies between facial muscle activity and self-reported emotion. Psychiatry Res. 95, 157-167. doi: 10.1016/S0165-1781(00)00168-2

Goldstein, M., Brendel, G., Tuescher, O., Pan, H., Epstein, J., Beutel, M., et al. (2007). Neural substrates of the interaction of emotional stimulus processing and motor inhibitory control: an emotional linguistic go/no-go fMRI study. Neuroimage 36, 1026-1040. doi: 10.1016/j.neuroimage.2007.01.056

Gross, J. J. (2001). Emotion regulation in adulthhood: timing is everything. Curr. Dir. Psychol. Sci. 10, 214-219. doi: 10.1111/1467-8721.00152

Hakamata, Y., Lissek, S., Bar-Haim, Y., Britton, J. C., Fox, N. A., Leibenluft, E., et al. (2010). Attention bias modification treatment: a meta-analysis toward the establishment of novel treatment for anxiety. Biol. Psychiatry 68, 982-990. doi: 10.1016/j.biopsych.2010.07.021

Hallion, L. S., and Ruscio, A. M. (2011). A meta-analysis of the effect of cognitive bias modification on anxiety and depression. Psychol. Bull. 137, 940-958. doi: $10.1037 / \mathrm{a} 0024355$

Holmes, A., Vuilleumier, P., and Eimer, M. (2003). The processing of emotional facial expression is gated by spatial attention: evidence from event-related brain potentials. Brain Res. Cogn. Brain Res. 16, 174-184. doi: 10.1016/S09266410(02)00268-9

Hopfinger, J. B., Buonocore, M. H., and Mangun, G. R. (2000). The neural mechanisms of top-down attentional control. Nat. Neurosci. 3, 284-291. doi: 10.1038/72999

Hum, K. M., Manassis, K., and Lewis, M. D. (2013). Neural mechanisms of emotion regulation in childhood anxiety. J. Child Psychol. Psychiatry 54, 552-564. doi: 10.1111/j.1469-7610.2012.02609.x

Hummer, T. A., Hulvershorn, L. A., Karne, H. S., Gunn, A. D., Wang, Y., and Anand, A. (2013). Emotional response inhibition in bipolar disorder: a functional magnetic resonance imaging study of trait- and state-related abnormalities. Biol. Psychiatry 73, 136-143. doi: 10.1016/j.biopsych.2012.06.036

Kropotov, J. D., Ponomarev, V. A., Hollup, S., and Mueller, A. (2011). Dissociating action inhibition, conflict monitoring and sensory mismatch into independent components of event related potentials in GO/NOGO task. Neuroimage 57, 565-575. doi: 10.1016/j.neuroimage.2011.04.060
Labuschagne, I., Phan, K. L., Wood, A., Angstadt, M., Chua, P., Heinrichs, M., et al. (2012). Medial frontal hyperactivity to sad faces in generalized social anxiety disorder and modulation by oxytocin. Int. J. Neuropsychopharmacol. 15, 883-896. doi: 10.1017/S1461145711001489

Linden, S. C., Jackson, M. C., Subramanian, L., Wolf, C., Green, P., Healy, D., et al. (2010). Emotion-cognition interactions in schizophrenia: implicit and explicit effects of facial expression. Neuropsychologia 48, 997-1002. doi: 10.1016/j.neuropsychologia.2009.11.023

Luo, W., Feng, W., He, W., Wang, N. Y., and Luo, Y. J. (2010). Three stages of facial expression processing: ERP study with rapid serial visual presentation. Neuroimage 49, 1857-1867. doi: 10.1016/j.neuroimage.2009.09.018

MacLeod, C., and Mathews, A. (2012). Cognitive bias modification approaches to anxiety. Annu. Rev. Clin. Psychol. 8, 189-217. doi: 10.1146/annurev-clinpsy032511-143052

Mantella, R. C., Butters, M. A., Dew, M. A., Mulsant, B. H., Begley, A. E., Tracey, B., et al. (2007). Cognitive impairment in late-life generalized anxiety disorder. Am. J. Geriatr. Psychiatry 15, 673-679. doi: 10.1097/JGP.0b013e31 $803111 \mathrm{f} 2$

Meyer, T., Qi, X. L., Stanford, T. R., and Constantinidis, C. (2011). Stimulus selectivity in dorsal and ventral prefrontal cortex after training in working memory tasks. J. Neurosci. 31, 6266-6276. doi: 10.1523/JNEUROSCI.6798-10.2011

Mohamed, T. N., Neumann, M. F., and Schweinberger, S. R. (2009). Perceptual load manipulation reveals sensitivity of the face-selective N170 to attention. Neuroreport 20, 782-787. doi: 10.1097/WNR.0b013e32832b7e24

Mulert, C., Pogarell, O., Juckel, G., Rujescu, D., Giegling, I., Rupp, D., et al. (2004). The neural basis of the $\mathrm{P} 300$ potential. Focus on the time-course of the underlying cortical generators. Eur. Arch. Psychiatry Clin. Neurosci. 254, 190-198. doi: 10.1007/s00406-004-0469-2

Nichols, T. E., and Holmes, A. P. (2002). Nonparametric permutation tests for functional neuroimaging: a primer with examples. Hum. Brain Mapp. 15, 1-25. doi: 10.1002/hbm.1058

Ochsner, K. N., and Gross, J. J. (2005). The cognitive control of emotion. Trends Cogn. Sci. 9, 242-249. doi: 10.1016/j.tics.2005.03.010

Ochsner, K. N., Ray, R. D., Cooper, J. C., Robertson, E. R., Chopra, S., Gabrieli, J. D., et al. (2004). For better or for worse: neural systems supporting the cognitive down- and up-regulation of negative emotion. Neuroimage 23, 483-499. doi: 10.1016/j.neuroimage.2004.06.030

Ofan, R. H., Rubin, N., and Amodio, D. M. (2013). Situation-based social anxiety enhances the neural processing of faces: evidence from an intergroup context. Soc. Cogn. Affect. Neurosci. 8, 1055-1061.

Pacheco-Unguetti, A. P., Acosta, A., Lupianez, J., Roman, N., and Derakshan, N. (2012). Response inhibition and attentional control in anxiety. Q. J. Exp. Psychol. (Hove) 65, 646-660. doi: 10.1080/17470218.2011.637114

Padmala, S., and Pessoa, L. (2010). Interactions between cognition and motivation during response inhibition. Neuropsychologia 48, 558-565. doi: 10.1016/j.neuropsychologia.2009.10.017

Pascual-Marqui, R. D. (2002). Standardized low-resolution brain electromagnetic tomography (sLORETA): technical details. Methods Find. Exp. Clin. Pharmacol. 24 Suppl D:5-12.

Paulus, M. P., and Yu, A. J. (2012). Emotion and decision-making: affect-driven belief systems in anxiety and depression. Trends Cogn. Sci. 16, 476-483. doi: 10.1016/j.tics.2012.07.009

Pergamin-Hight, L., Naim, R., Bakermans-Kranenburg, M. J., van, I. M. H., and Bar-Haim, Y. (2014). Content specificity of attention bias to threat in anxiety disorders: a meta-analysis. Clin. Psychol. Rev. 35C, 10-18.

Peschard, V., Philippot, P., Joassin, F., and Rossignol, M. (2013). The impact of the stimulus features and task instructions on facial processing in social anxiety: an ERP investigation. Biol. Psychol. 93, 88-96. doi: 10.1016/j.biopsycho.2013.01.009

Pessoa, L. (2009). How do emotion and motivation direct executive control? Trends Cogn. Sci. 13, 160-166. doi: 10.1016/j.tics.2009.01.006

Pessoa, L., Padmala, S., and Morland, T. (2005). Fate of unattended fearful faces in the amygdala is determined by both attentional resources and cognitive modulation. Neuroimage 28, 249-255. doi: 10.1016/j.neuroimage.2005.05.048

Pizzagalli, D. A., Oakes, T. R., Fox, A. S., Chung, M. K., Larson, C. L., Abercrombie, H. C., et al. (2004). Functional but not structural subgenual prefrontal cortex abnormalities in melancholia. Mol. Psychiatry 9, 393-405. doi: 10.1038/sj.mp.4001469 
Righart, R., and de Gelder, B. (2007). Impaired face and body perception in developmental prosopagnosia. Proc. Natl. Acad. Sci. U.S.A. 104, 17234-17238. doi: 10.1073/pnas.0707753104

Righi, S., Mecacci, L., and Viggiano, M. P. (2009). Anxiety, cognitive self-evaluation and performance: ERP correlates. J. Anxiety Disord. 23, 1132-1138. doi: 10.1016/j.janxdis.2009.07.018

Roemer, L., Lee, J. K., Salters-Pedneault, K., Erisman, S. M., Orsillo, S. M., and Mennin, D. S. (2009). Mindfulness and emotion regulation difficulties in generalized anxiety disorder: preliminary evidence for independent and overlapping contributions. Behav. Ther. 40, 142-154. doi: 10.1016/j.beth.2008.04.001

Rossignol, M., Philippot, P., Douilliez, C., Crommelinck, M., and Campanella, S. (2005). The perception of fearful and happy facial expression is modulated by anxiety: an event-related potential study. Neurosci. Lett. 377, 115-120. doi: 10.1016/j.neulet.2004.11.091

Russell, J. A. (1989). Affect grid: a single-item scale of pleasure and arousal. J. Pers. Soc. Psychol. 57, 493-502. doi: 10.1037/0022-3514.57.3.493

Salters-Pedneault, K., Roemer, L., Tull, M. T., Rucker, L., and Mennin, D. S. (2006). Evidence of broad deficits in emotion regulation associated with chronic worry and generalized anxiety disorder. Cogn. Ther. Res. 30, 469-480. doi: 10.1007/s10608-006-9055-4

Sass, S. M., Heller, W., Stewart, J. L., Silton, R. L., Edgar, J. C., Fisher, J. E., et al. (2010). Time course of attentional bias in anxiety: emotion and gender specificity. Psychophysiology 47, 247-259. doi: 10.1111/j.1469-8986.2009.00926.x

Schneider, F., Gur, R. C., Gur, R. E., and Muenz, L. R. (1994). Standardized mood induction with happy and sad facial expressions. Psychiatry Res. 51, 19-31. doi: 10.1016/0165-1781(94)90044-2

Schonfelder, S., Kanske, P., Heissler, J., and Wessa, M. (2013). Time course of emotion-related responding during distraction and reappraisal. Soc. Cogn. Affect. Neurosci. 9, 1310-1319. doi: 10.1093/scan/nst116

Sehlmeyer, C., Konrad, C., Zwitserlood, P., Arolt, V., Falkenstein, M., and Beste, C. (2010). ERP indices for response inhibition are related to anxiety-related personality traits. Neuropsychologia 48, 2488-2495. doi: 10.1016/j.neuropsychologia.2010.04.022

Semlitsch, H. V., Anderer, P., Schuster, P., and Presslich, O. (1986). A solution for reliable and valid reduction of ocular artifacts, applied to the P300 ERP. Psychophysiology 23, 695-703. doi: 10.1111/j.1469-8986.1986.tb00696.x

Shafritz, K. M., Collins, S. H., and Blumberg, H. P. (2006). The interaction of emotional and cognitive neural systems in emotionally guided response inhibition. Neuroimage 31, 468-475. doi: 10.1016/j.neuroimage.2005.11.053

Shapiro, K., Hillstrom, A. P., and Husain, M. (2002). Control of visuotemporal attention by inferior parietal and superior temporal cortex. Curr. Biol. 12, 1320-1325. doi: 10.1016/S0960-9822(02)01040-0

Snodgrass, J. G., and Corwin, J. (1988). Perceptual identification thresholds for 150 fragmented pictures from the Snodgrass and Vanderwart picture set. Percept. Mot. Skills 67, 3-36. doi: 10.2466/pms.1988.67.1.3

Stanislaw, H., and Todorov, N. (1999). Calculation of signal detection theory measures. Behav. Res. Methods Instrum. Comput. 31, 137-149. doi: 10.3758/BF03207704

Taylor, S. F., Phan, K. L., Decker, L. R., and Liberzon, I. (2003). Subjective rating of emotionally salient stimuli modulates neural activity. Neuroimage 18, 650-659. doi: 10.1016/S1053-8119(02)00051-4

Termine, N. T., and Izard, C. E. (2009). Infants' responses to their mothers expressions of joy and sadness. Dev. Psychol. 66, 223-229.
Valdes-Conroy, B., Aguado, L., Fernandez-Cahill, M., Romero-Ferreiro, V., and Dieguez-Risco, T. (2014). Following the time course of face gender and expression processing: a task-dependent ERP study. Int. J. Psychophysiol. 92, 59-66. doi: 10.1016/j.ijpsycho.2014.02.005

Veit, R., Flor, H., Erb, M., Hermann, C., Lotze, M., Grodd, W., et al. (2006). Brain circuits involved in emotional learning in antisocial behavior and social phobia in humans. Neurosci. Lett. 328, 233-236. doi: 10.1016/S0304-3940(02) 00519-0

Verbruggen, F., Aron, A. R., Stevens, M. A., and Chambers, C. D. (2010). Theta burst stimulation dissociates attention and action updating in human inferior frontal cortex. Proc. Natl. Acad. Sci. U.S.A. 107, 13966-13971. doi: 10.1073/pnas.1001957107

Vitacco, M. J., Neumann, C. S., Robertson, A. A., and Durrant, S. L. (2002). Contributions of impulsivity and callousness in the assessment of adjudicated male adolescents: a prospective study. J. Pers. Assess. 78, 87-103. doi: 10.1207/S15327752JPA7801_06

Vuilleumier, P., and Pourtois, G. (2007). Distributed and interactive brain mechanisms during emotion face perception: evidence from functional neuroimaging. Neuropsychologia 45, 174-194. doi: 10.1016/j.neuropsychologia.2006. 06.003

Williams, L. M., Palmer, D., Liddell, B. J., Song, L., and Gordon, E. (2006). The 'when' and 'where' of perceiving signals of threat versus non-threat. Neuroimage 31, 458-467. doi: 10.1016/j.neuroimage.2005.12.009

Winston, J. S., O’Doherty, J., and Dolan, R. J. (2003). Common and distinct neural responses during direct and incidental processing of multiple facial emotions. Neuroimage 20, 84-97. doi: 10.1016/S1053-8119(03)00303-3

Winton, E. C., Clark, D. M., and Edelmann, R. J. (1995). Social anxiety, fear of negative evaluation and the detection of negative emotion in others. Behav. Res. Ther. 33, 193-196. doi: 10.1016/0005-7967(94)E0019-F

Wittchen, H. U. (2002). Generalized anxiety disorder: prevalence, burden, and cost to society. Depress. Anxiety 16, 162-171. doi: 10.1002/da.10065

Yu, F., Ye, R., Sun, S., Carretie, L., Zhang, L., Dong, Y., et al. (2014). Dissociation of neural substrates of response inhibition to negative information between implicit and explicit facial Go/Nogo tasks: evidence from an electrophysiological study. PLoS ONE 9:e109839. doi: 10.1371/journal.pone.0109839

Yu, F., Yuan, J., and Luo, Y. J. (2009). Auditory-induced emotion modulates processes of response inhibition: an event-related potential study. Neuroreport 20 , 25-30. doi: 10.1097/WNR.0b013e32831ac9b1

Yuan, J., He, Y., Qinglin, Z., Chen, A., and Li, H. (2008). Gender differences in behavioral inhibitory control: ERP evidence from a two-choice oddball task. Psychophysiology 45, 986-993. doi: 10.1111/j.1469-8986.2008.00693.x

Conflict of Interest Statement: The authors declare that the research was conducted in the absence of any commercial or financial relationships that could be construed as a potential conflict of interest.

Copyright (c) 2015 Yu, Zhu, Zhang, Chen, Li, Zhang, Ye, Dong, Luo, Hu and Wang. This is an open-access article distributed under the terms of the Creative Commons Attribution License (CC BY). The use, distribution or reproduction in other forums is permitted, provided the original author(s) or licensor are credited and that the original publication in this journal is cited, in accordance with accepted academic practice. No use, distribution or reproduction is permitted which does not comply with these terms. 